\title{
Proof of Four Color Map Theorem by Using PRN of Graph \\ ${ }^{1}$ Mr. H. R. Bhapkar, ${ }^{2}$ Dr. J. N. Salunke \\ ${ }^{1}$ Asstt. Prof. in Mathematics, Sinhgad Technical Education Society's, Smt. Kashibai Navale College of Engg. Vadgaon, Pune 41. \\ ${ }^{2}$ Director, School of Mathematical Sciences, Swami Ramanand Teerth \\ Marathwada University, Nanded.
}

Keywords: PRN of Graph, Four Color Map Theorem.

ABSTRACT. This paper intends to study the relation between PRN and chromatic number of planar graphs. In this regard we investigate that isomorphic or 1 isomorphic graph may or may not have equal PRN and few other related results. Precisely, we give simple proof of Four Color Map Theorem.

\section{INTRODUCTION}

In this section, we present a brief survey of those results of graph theory, which we shall need later. The reader is referred to $[6,7,8,10]$ for a fuller treatment of the subject.

\subsection{Graphs:}

A graph $G$ is an ordered pair $(V(G), E(G))$ where i) $V(G)$ is a non empty finite set of elements, known as vertices. $V(G)$ is known as vertex set. ii) $E(G)$ is a family of unordered pairs ( not necessarily distinct ) of elements of $\mathrm{V}$, known as edges of $\mathrm{G}$. $\mathrm{E}(\mathrm{G})$ is known as Edge set.

Each vertex of graph $G$ is represented by a point or small circle in the plane. Every edge is represented by a continuous arc or straight line segment. A certain pairs of vertices of graph are joined by two or more edges, such edges are known as multiple or parallel edges. An edge joining a vertex to itself, is called a loop. A graph without loops or multiple edges is called a simple graph. Non-simple graphs are known as multiple graphs. The degree or valency of a vertex $\mathrm{v}$ of graph $\mathrm{G}$ is the number of edges incident at that $v$. It is denoted by $d(v)$. A vertex of degree 1 is called a pendent vertex. A vertex of zero degree is said to be isolated vertex. An edge, whose one end vertex is a pendant vertex, is known as pendant edge.

A walk of a graph is defined as a finite alternating sequence of vertices and edges, beginning and ending with vertices, such that each edge is incident with the vertices preceding and following it. Vertices of graph with which a walk begins and ends, are called its terminal vertices. A walk, in which terminal vertices are same, is called as the closed walk .Otherwise open walk. A graph $G$ is said to be the connected graph if there exists at least one walk between every pair of vertices in $\mathrm{G}$.Otherwise graph $\mathrm{G}$ is disconnected. The vertex connectivity of a connected graph $\mathrm{G}$ is defined as the minimum number of vertices whose removal from $G$ leaves the remaining graph disconnected. A connected graph is said to be 2- connected if its vertex connectivity is two. [7]

1.2 Fat $K_{n}$ : A complete graph $K n$ is said to be fat complete graph on $n$ vertices $\left(\right.$ Fat $\left.K_{n}\right)$ if there exists at least one parallel edge between vertices of Kn.

1.3 Isomorphism: A graph $G_{1}\left(V_{1}, E_{1}\right)$ is said to be isomorphic to the graph $G 2\left(V_{2}, E_{2}\right)$ if

i) There is a one to one correspondence between the vertex sets $V_{1}$ and $V_{2}$.

ii) There is a one to one correspondence between the edge sets E1 and $E_{2}$ in such a way that if el is an edge with end vertices $u_{1}$ and $v_{1}$ in $G_{1}$ then the corresponding edge $e_{2}$ in $G_{2}$ has its end points the vertices $\mathrm{u}_{2}$ and $\mathrm{v}_{2}$ in $\mathrm{G}_{2}$ which correspond to $\mathrm{u}_{1} \& \mathrm{v}_{1}$ respectively. 
Such a pair of correspondence is called a graph isomorphism.

1.4 Separable graph: A connected graph $\mathrm{G}$ is said to be separable if its vertex connectivity is one. All other connected graphs are called nonseparable graphs. A connected graph $G$ is said to be separable if there exists a subgraph $G_{1}$ in $G$ such that $G\left(\right.$ the complement of $G_{1}$ in $G$ ) and $G 1$ have only one vertex in common.

1.5 1-isomorphism: Two graphs $G_{1}$ and $G_{2}$ are said to be 1- isomorphic graphs if they become isomorphic to each other under repeated application of the following operation 1.

Operation1: Split a cut vertex of graph into two vertices to produce two disjoint subgraphs. Therefore, two graphs $G_{1}$ and $G_{2}$ are said to be 1-isomorphic graphs if and only if the blocks or components of the graph $\mathrm{G}_{1}$ are isomorphic to the blocks or components of the graph $\mathrm{G}_{2}$. From this definition, it is clear that two non-separable graphs are 1 isomorphic iff they are isomorphic. [7]

\section{PLANAR GRAPHS}

2.1 Planar Graph: A graph $G$ is a planar graph if it is possible to represent it in the plane such that no two edges of the graph intersect except possibly at a vertex to which they are both incident. Any such drawing of planar graph $\mathrm{G}$ in a plane is a planar embedding of $\mathrm{G}$.

If $x$ any point in the plane of a planar graph that is neither a vertex nor a point on an edge, the set of all points in the plane that can be reached from $\mathrm{x}$ by traversing along a curve that does not have a vertex of the graph or a point of an edge as an intermediate point, is the region of the graph that contains $\mathrm{x}$. Thus the plane graph $\mathrm{G}$ partitions the plane into the different regions of $\mathrm{G}$. Among these regions there is exactly one region whose area is not finite, is called exterior or infinite region. Every other region is an interior region. The boundary of a region is a sub-graph formed by the vertices and edges encompassing that region. If the boundary of the exterior region of a planar graph is a cycle, that cycle is known as the maximal cycle of that graph. The degree of the region is the number of edges in a closed walk that encloses it. The region formed by three edges is known as triangular region. The region formed by four edges is known as rectangular region. [6,7]

Theorem 2.1: If a connected planar graph of $n$ vertices, $m$ edges has $f$ regions or faces, then $n-m+$ $\mathrm{f}=2$

2.2 Geometric Dual: Let $G$ be a plane graph with $n$ Regions or faces say $R_{1}, R_{2}, R_{3}, \ldots R_{n}$. Let us place points ( say vertices ) $V_{1}, V_{2}, V_{3}, \ldots V_{n}$, one in each of the regions. Let us join these vertices $\mathrm{V}_{\mathrm{i}}$ according to the following procedure.

i) If two regions $\mathrm{Ri}$ and $\mathrm{Rj}$ are adjacent then draw a line joining vertices $\mathrm{Vi}$ and $\mathrm{Vj}$ that intersect the common edge between $\mathrm{Ri}$ and $\mathrm{Rj}$ exactly once.

ii) If there are two or more edges common between $\mathrm{Ri}$ and $\mathrm{Rj}$, then draw one line between vertices $\mathrm{Vi}$ and $\mathrm{Vj}$ for each of the common edges.

iii) For an edge 'e' lying entirely in one region say $\mathrm{Ri}$, draw a self loop at pendant vertex $\mathrm{Vi}$ intersecting e exactly once.

By this procedure, we obtain a new graph $G^{*}$ consisting of $V_{1}, V_{2}, V_{3}, \ldots V_{n}$ vertices and edges joining these vertices. Such a graph $\mathrm{G}^{*}$ is called a geometric dual of $G$ (a dual of G). [7]

Theorem 2.2: The geometric dual of a planar graph is planar.

2.3 * isomorphism: Two graphs are said to be *isomorphic if their geometric duals are isomorphic.

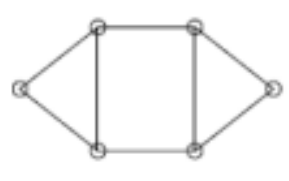

$\mathrm{G}_{1}$

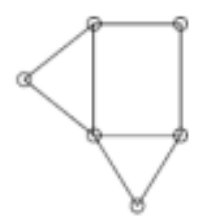

$\mathrm{G}_{2} \quad$ Fig.2.1

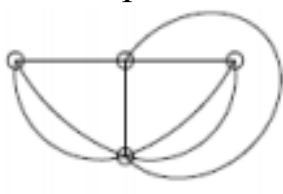

$\mathrm{G}_{1}$ *

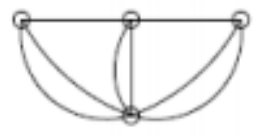

$\mathrm{G}_{2}{ }^{*}$

Every graph is * isomorphic to itself. The definition is symmetric, and if $\mathrm{G}_{1}$ and $\mathrm{G}_{2}$ are *isomorphic to $\mathrm{G}_{2}$ and $\mathrm{G}_{3}$, respectively, then $\mathrm{G}_{1}$ and $\mathrm{G}_{3}$ are * isomorphic. Thus *isomorphism is an equivalence relation. Suppose two graphs are * isomorphic, then it is clear that the two graphs must have same 
number of edges and same number of regions formed by equal number of edges. There is no any condition on number of vertices of the two graphs. Thus these conditions are necessary for two graphs to be *isomorphic. However, these conditions are not sufficient. [4]

2.4 Four Color Map Problem: Planar map is a set of pairwise disjoint subsets of the plane, known as regions of the map. Two regions a map are adjacent if they have a common boundary that is not a corner. A vertex or point of a map is said to be corner if it is a common point of three or more regions. A coloring of a graph is an assignment of colors to its vertices (or regions) so that no two adjacent vertices (or regions) have the same color. The set of all vertices (or regions) with same color in graph, is called a color class. [1]

Theorem 2.3: (Four Color Map Theorem) Every planar map can be colored with four or fewer colors.

The Four Color Conjecture was first stated 200 years ago and finally proved conclusively in 1976. The professor of mathematics, Augustus De Morgan (1806-71) and his friend William Rowan Hamilton studied this theorem and gave first proof. In 1879, Alfred Kempe, published a short paper on coloring of maps. He added some other ideas of coloring. In 1879, Alfred Kempe published this proof in the American Journal of Mathematics in simple versions. In 1980, Tait P.G. offered independent solution to this problem. After collaborating with John Koch on the problem of reducibility, in 1976, Kenneth Appel and Wolfgang Haken gave the complete proof to the four color

conjecture by reducing the testing problem to an unavoidable set with 1936 configurations. Because of the computer based proof, many Mathematicians were not agreeing with this proof. However, many proofs written by different Mathematicians have been found to be faulty. So all we have been waiting for the simple proof of this theorem. [2, 3, 4]

2.5 Pivot Region of graph: A region or face $\mathrm{R}$ of a planar graph is said to be a pivot region of graph if all other regions of graph are adjacent to R. Every region of a complete graph on four vertices $\left(\mathrm{K}_{4}\right)$ is a pivot region. So $\mathrm{K}_{4}$ has four pivot regions. The number of pivot regions of a planar graph is known as Pivot Region Number of that Graph. It is denoted by PRN (G). There are graphs which have PRN equal to $1,2,3$ and 4 . PRN $\left(\mathrm{K}_{4}\right)=4$. [5]

2.6 HB Graph: A planar graph is said to be HB graph if it has a pivot region. Actually such graphs should name as Neighborhood graphs, but for convenience, we select two continue letters from that name. A planar graph with only one pivot region is known as Uni-HB graph. A graph having two or more pivot regions is called Multi-HB graph. A HB graph, in which every region is a pivot region of that graph, is called Complete HB graph. The Wheel graph on 5 vertices is an Uni-HB graph. The complete graphs on vertices 1,2,3,4 are complete HB graphs. [5]

\section{THE MAIN RESULTS}

Theorem 1: If $\mathrm{G}$ is any planar graph then PRN $(\mathrm{G})$ is at most 4.

Proof: The graphs $\mathrm{K}_{1}, \mathrm{~K}_{3}, \mathrm{~K}_{4}-\{\mathrm{e}\}$, and $\mathrm{K}_{4}$ have respectively PRN 1, 2, 3 and 4. Suppose graph $\mathrm{G}$ has PRN 5. So there exist at least five regions which are adjacent to each other. Let us place one vertex in each region. In the geometric dual of $\mathrm{G}$, at least five vertices are adjacent to each other, which form a complete graph on 5 vertices, $\mathrm{K}_{5}$. So $\mathrm{K}_{5}$ is a subgraph of $\mathrm{G}^{*}$. But graph $\mathrm{K}_{5}$ is not planar graph. Therefore graph $\mathrm{G}^{*}$ is not planar graph, which is contradiction. Hence the proof.

Theorem 2: If $G$ is a planar graph, then $P R N(G) \leq C R(G)$.

Proof: Let $G$ be a planar graph on $n$ vertices and $f$ faces or regions. Suppose PRN $(G)=p$ and $C R$ $(G)=c$. There are $p$ faces which are adjacent to each other. So the chromatic number of $G$ is at least p. Thus PRN $(\mathrm{G})=\mathrm{p} \leq \mathrm{CR}(\mathrm{G})$.

Alternately, if $\mathrm{CR}(\mathrm{G})=\mathrm{c}$, then there are at most $\mathrm{c}$ regions which are adjacent to each other. So $\operatorname{PRN}(\mathrm{G}) \leq \mathrm{c}=\mathrm{CR}(\mathrm{G})$

Theorem 3: If $G$ is a complete $H B$ graph $G$ then $P R N(G)=C R(G)$.

Proof: Let $G$ be a complete HB graph on $n$ vertices and $f$ faces. All faces of $G$ are adjacent to each other. Therefore PRN $(\mathrm{G})=\mathrm{f}$ and $\mathrm{CR}(\mathrm{G})=\mathrm{f}$. Thus PRN $(\mathrm{G})=\mathrm{CR}(\mathrm{G})$. 
Theorem 4: If $G_{1}$ and $G_{2}$ are * isomorphic graphs then $P R N\left(G_{1}\right)=\operatorname{PRN}\left(G_{2}\right)$.

Proof: By the definition of * isomorphic graphs, $\mathrm{G}_{1}$ and $\mathrm{G}_{2}$ have region correspondence. Thus PRN $\left(\mathrm{G}_{1}\right)=\operatorname{PRN}\left(\mathrm{G}_{2}\right)$.

Theorem 5: If $G_{1}$ and $G_{2}$ are * isomorphic graphs then $C R\left(G_{1}\right)=C R\left(G_{2}\right)$.

Proof: Graphs $G_{1}$ and $G_{2}$ are * isomorphic. So $G_{1}$ and $G_{2}$ have region correspondence. Thus $C R$ $\left(\mathrm{G}_{1}\right)=\mathrm{CR}\left(\mathrm{G}_{2}\right)$.

Theorem 6: If $\mathrm{G}_{1}$ and $\mathrm{G}_{2}$ are * isomorphic graphs then $P R N\left(\mathrm{G}_{1}\right)$ and $C R\left(\mathrm{G}_{2}\right)$ may or may not be equal.

Proof: Let $\mathrm{G}_{1}$ and $\mathrm{G}_{2}$ be two * isomorphic graphs as well as complete HB graphs. By theorem 3, $\operatorname{PRN}\left(\mathrm{G}_{1}\right)$

and $C R\left(G_{2}\right)$ are equal. Suppose $G_{3}$ and $G_{4}$ are two * isomorphic graphs, which are given below.

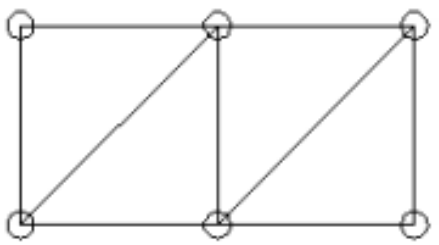

$\mathrm{G}_{3}$

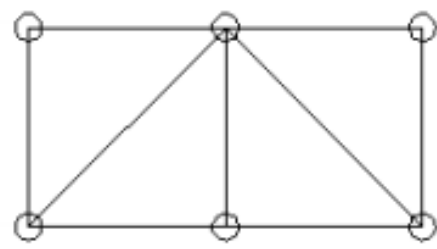

$\mathrm{G}_{4}$

Fig. 3.1

The PRN of graph $G_{3}$ is 1 and the chromatic number of $G_{4}$ is 3 . Thus $P R N\left(G_{3}\right) \neq C R\left(G_{4}\right)$.

Theorem 7: Two isomorphic graphs may or may not have equal PRN.

Proof: Let $G_{1}, G_{2}$ and $G_{3}$ be three mutually isomorphic graphs. Graphs $G_{1}$ and $G_{2}$ have only one pivot region, so they have equal PRN.

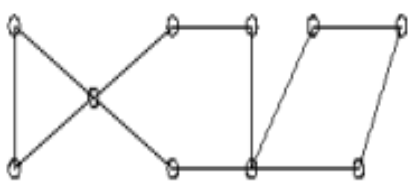

$\mathrm{G}_{1}$

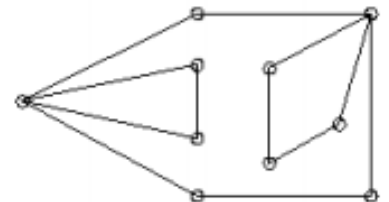

$\mathrm{G}_{2}$

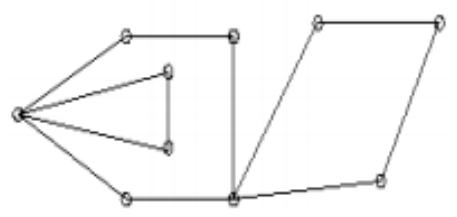

$\mathrm{G}_{3}$

Fig. 3.2

Graph $\mathrm{G}_{3}$ has no pivot region, so it has no PRN. Therefore two isomorphic graphs may or may not have equal PRN.

Theorem 8: Two 1 isomorphic graphs may or may not have equal PRN.

Proof: Let $G_{1}, G_{2}$ and $G_{3}$ be three mutually 1 isomorphic graphs. Graphs $G_{1}$ and $G_{2}$ have only one pivot region, but $\mathrm{G}_{3}$ has no pivot region. So PRN $\left(\mathrm{G}_{1}\right)=1, \operatorname{PRN}\left(\mathrm{G}_{2}\right)=1, \operatorname{PRN}\left(\mathrm{G}_{3}\right)=0$. Thus two 1 isomorphic graphs may or may not have equal PRN.

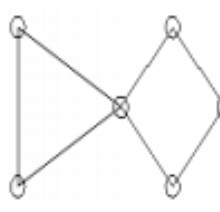

$\mathrm{G}_{1}$

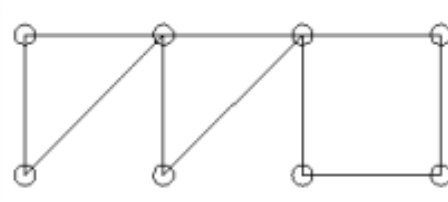

$\mathrm{G}_{2}$

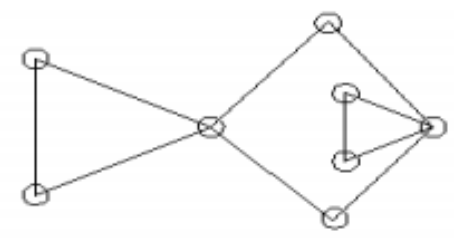

$\mathrm{G}_{3}$

Fig. 3.3

Theorem 9: Every complete HB graph can be colored in four or fewer colors.

Proof: By theorem 1, if $G$ is any planar graph then PRN $(G)$ is at most 4 . By theorem 3, if $G$ is a complete HB graph $G$ then PRN $(G)=C R(G)$. Therefore every complete HB graph is four colorable. 
Theorem 10: (The Four Color Theorem) Every planar graph (map) can be colored in four or fewer colors.

Proof: The planar graphs $\mathrm{K}_{2}, \mathrm{~K}_{3}, \mathrm{~K}_{4}-\{\mathrm{e}\}$, and $\mathrm{K}_{4}$ have respectively 1, 2, 3 and 4 chromatic numbers. Suppose $\mathrm{G}$ is a planar graph with $\mathrm{CR}(\mathrm{G})=5$. So there are only two possibilities

Case 1: There are five regions in $G$, which are adjacent to each other. Let us put one vertex in each region of $\mathrm{G}$. Find geometric dual of $\mathrm{G}$, say $\mathrm{G}^{*}$. In $\mathrm{G}^{*}$, five vertices are adjacent to each other, which forms $\mathrm{K}_{5}$.

Therefore $\mathrm{K}_{5}$ is the subgraph of $\mathrm{G}^{*}$. So $\mathrm{G}^{*}$ is not planar graph, which is the contradiction.

Case 2: If not all regions of $G$ are adjacent to each other, then find geometric dual of $G$, say $G^{*}$. In $\mathrm{G}^{*}$, make partitions of vertices such that two vertices in $\mathrm{G}^{*}$ belong to same partition if and only if corresponding regions in $\mathrm{G}$ have same color. So there are five partitions only say $\mathrm{P}_{1}, \mathrm{P}_{2}, \mathrm{P}_{3}, \mathrm{P}_{4}$ and $\mathrm{P}_{5}$. No vertices of the same partition are adjacent to each other. Construct new graph $\mathrm{G} 1$ from $\mathrm{G}^{*}$ by the following procedure.

i) Let $P_{1}, P_{2}, P_{3}, P_{4}$ and $P_{5}$ be the vertices of $G_{1}$

$\mathrm{P}_{i}$ and $\mathrm{P}_{\mathrm{j}}$ are adjacent if and only if at least one vertex in $\mathrm{Pi}$ is adjacent to at least one vertex of $\mathrm{P}_{\mathrm{j}}$ for $i \neq j$.

ii) All $\mathrm{Pi}$ and $\mathrm{Pj}$, for $\mathrm{i} \neq \mathrm{j}$ are adjacent to each other.

This new graph is similar to fat $\mathrm{K}_{5}$, which is the contradiction to the fact that $\mathrm{G}^{*}$ is planar graph.

Theorem 11: Every tree has PNR 1.

Proof: Every tree on any number of vertices has only one region. So every tree has PRN 1.

\section{REFERENCES}

[1] Appel K. and W. Haken, Every Planar Map is Four Colorable, Bulletin of American Mathematical society, 82 (1977), 711-712.

[2] Appel K. and W. Haken, Every Planar Map is Four Colorable, Contemporary Mathematics 98, American Mathematical society, 1989

[3] Brooks R.L., On Coloring of Nodes of a Network, Proc. Cambridge Phil. Society, Vol. 37, 1941, 194-197.

[4] H. R. Bhapkar and J. N. Salunke, *isomorphism of graphs, in International Journal of Mathematical Sciences and Engineering Applications, Vol. 8, No. II, 0973-9424, March 14.

[5] H. R. Bhapkar and J. N. Salunke, The Geometric Dual of HB Graph, *outerplanar Graph and Related Aspects, in Bulletin of Mathematical Sciences and Applications, ISSN 2278-9634, Volume 3, No. 3, pp 114-119, August 2014.

[6] Harary, F. Graph Theory. Reading, MA: Addison-Wesley, pp. 113-115, 1994.

[7] Narsingh Deo, Graph Theory with Applications To Engineering and Computer Science, Prentice -Hall of India,2003,88-111.

[8] Robin J. Wilson, Introduction to Graph Theory, Pearson, 978-81-317-0698-5, 2011

[9] Tait P.G., On the coloring of maps, Proceeding Royal Society, Edinburgh Sect. A 10 (18781810), 501-503,729

[10] V. K. Balakrishnan, Schaum's outline of theory and problems of graph theory, 198-243, 2008. 